\title{
A note on causal and CITE iterative learning control algorithms
}

\author{
Mikael Norrlöf, Svante Gunnarsson \\ Division of Automatic Control \\ Department of Electrical Engineering \\ Linköpings universitet, SE-581 83 Linköping, Sweden \\ WWW: http://WwW. control.isy.liu.se \\ E-mail: mino@isy.liu.se, svante@isy.liu.se
}

4th June 2003

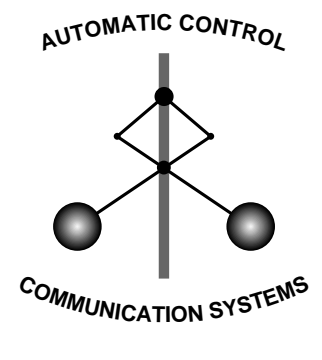

LINKÖPING

Report no.: LiTH-ISY-R-2510

Submitted to Automatica

Technical reports from the Control \& Communication group in Linköping are available at http://www. control.isy.liu.se/publications. 


\begin{abstract}
Time and frequency domain convergence properties of causal and Current Iteration Tracking Error (CITE) discrete time Iterative Learning Control (ILC) algorithms are discussed. Considering necessary and sufficient convergence conditions basic matrix properties can be utilized to show that causal as well as CITE ILC algorithms converge to zero error in only very restrictive special cases. The frequency domain convergence conditions, sufficient for monotone convergence, are studied using a discrete-time version of Bode's integral theorem. The result is that causal and CITE ILC algorithms will not satisfy the frequency domain conditions except if the system has relative degree zero or it is accepted that the algorithms do not converge to zero error.
\end{abstract}

Keywords: iterative learning control, frequency domain, causal 


\title{
A note on causal and CITE iterative learning control algorithms
}

\author{
Mikael Norrlöf and Svante Gunnarsson* \\ Department of Electrical Engineering, Linköping University, SE-58183 Linköping,
} Sweden

\begin{abstract}
Time and frequency domain convergence properties of causal and Current Iteration Tracking Error (CITE) discrete time Iterative Learning Control (ILC) algorithms are discussed. Considering necessary and sufficient convergence conditions basic matrix properties can be utilized to show that causal as well as CITE ILC algorithms converge to zero error in only very restrictive special cases. The frequency domain convergence conditions, sufficient for monotone convergence, are studied using a discrete-time version of Bode's integral theorem. The result is that causal and CITE ILC algorithms will not satisfy the frequency domain conditions except if the system has relative degree zero or it is accepted that the algorithms do not converge to zero error.
\end{abstract}

Key words: Iterative Learning Control, frequency domain, causal

* Corresponding author. Fax:+46-13-282622; Tel: $+46-13-281747$
Email address: mino, svante@isy.liu.se (Mikael Norrlöf and Svante

Gunnarsson). 


\section{Introduction}

Iterative Learning Control (ILC) has proven to be a competitive control method in many applications, and the most well known is probably the robotics domain. Some examples are given in Arimoto et al. (1984); Horowitz (1993); Lange and Hirzinger (1999); Norrlöf and Gunnarsson (2002a). The classical formulation of the ILC problem is to use an iterative procedure to find the input to a system such that the output follows a desired output as well as possible. Traditionally the ILC input signal is formed using the error from previous iterations, i.e. the input $u_{k+1}$ is computed using $e_{k}$. Recently it has been proposed, see e.g. Chen et al. (1996a,b) and French et al. (1999), to use also the current error, $e_{k+1}$, when forming $u_{k+1}$. Since this approach necessarily leads to a causal relationship between the error and the input signal it is closely related to causal ILC algorithms which recently have been studied in Goldsmith (2002).

The aim of this paper is to investigate some aspects of CITE and casual ILC algorithms. Some initial results were presented in Norrlöf and Gunnarsson (2002b) and the goal is to give a more complete treatment here. The analysis is carried out in discrete time since in practice the implementation of an ILC algorithm has to be done in discrete time. Both time domain and frequency domain convergence properties are considered. The time domain approach leads to a necessary and sufficient convergence condition, which is expressed in terms of eigenvalues of a particular matrix. For practical reasons, mainly monotone convergence, a sufficient convergence condition expressed using singular values is of greater interest. This condition can then be tied to a frequency domain condition involving the magnitude of a transfer function. It is also discussed how a requirement on zero asymptotic error affects the possibilities to obtain convergence. The main result is that convergence to zero error using a causal ILC algorithm can not be obtained in problems of practical interest. One way 
out of the problem is to accept a non-zero final error, and a second way is to use a non-causal ILC algorithm.

Section 2 gives a brief description of the kind of systems and ILC algorithms that are considered, and the fundamental convergence conditions are presented. In Sections 3 and 4 the time and frequency domain convergence properties are investigated. Section 5 contains a numerical illustration, and finally Section 6 contains some conclusions.

\section{System and algorithm description}

\subsection{System}

The paper deals with linear systems that operate in discrete time during finite time intervals. A system can then be represented in a matrix form,

$$
\boldsymbol{y}_{k}=\boldsymbol{T}_{u} \boldsymbol{u}_{k}
$$

where

$$
\boldsymbol{y}_{k}=\left(y_{k}(0), \ldots, y_{k}(n-1)\right)^{T}, \quad \boldsymbol{u}_{k}=\left(u_{k}(0), \ldots, u_{k}(n-1)\right)^{T}
$$

and $\boldsymbol{T}_{u}$ is a a Toeplitz matrix

$$
\boldsymbol{T}_{u}=\left(\begin{array}{cccc}
g_{T_{u}}(0) & 0 & \ldots & 0 \\
g_{T_{u}}(1) & g_{T_{u}}(0) & & \vdots \\
\vdots & & \ddots & 0 \\
g_{T_{u}}(n-1) & g_{T_{u}}(n-2) & \ldots & g_{T_{u}}(0)
\end{array}\right)
$$

Here $g_{T_{u}}(t), t \in[0, n-1]$ is the impulse response of the system, the sampling time is assumed to be 1 , and $n$ is the number of samples. In equations (1) 
and (3) it is also assumed that the system description is $k$-independent. If the system is linear time variant, the matrix $\boldsymbol{T}_{u}$ does not become a lower triangular Toeplitz matrix but instead a general lower triangular matrix. When the system is time invariant it can be described using transfer operators

$$
y_{k}(t)=T_{u}(q) u_{k}(t)
$$

where

$$
T_{u}(q)=\sum_{k=0}^{\infty} g_{T_{u}}(k) q^{-k}
$$

The symbols describing the vectors and matrices in the matrix description in equation (1) to equation (3) are throughout the paper given in bold face to make it easier to distinguish between the representation in (4) and the matrix description.

\subsection{Algorithms}

Analogous to the system description above the ILC algorithms can also be formulated using both matrices and transfer operators. In the matrix formulation the algorithm is given by

$$
\boldsymbol{u}_{k+1}=\boldsymbol{Q}\left(\boldsymbol{u}_{k}+\boldsymbol{L} \boldsymbol{e}_{k}+\boldsymbol{L}_{c} \boldsymbol{e}_{k+1}\right)
$$

where the matrices $\boldsymbol{Q}, \boldsymbol{L}$ and $\boldsymbol{L}_{c}$ are design variables. In equation (6) $\boldsymbol{e}_{k}$ denotes the difference between the desired output and the actual output, i.e.

$$
\boldsymbol{e}_{k}=\boldsymbol{r}-\boldsymbol{y}_{k}
$$

In a causal ILC algorithm the matrix $\boldsymbol{L}$ is restricted to be lower triangular, but in a non-causal ILC algorithm it has non-zero elements above the diagonal. Since the matrix $\boldsymbol{L}_{c}$ operates on the current error it has to be lower triangular since only causal operations can be carried out. 
Using transfer operators the ILC algorithm is described by

$$
u_{k+1}(t)=Q(q)\left(u_{k}(t)+L(q) e_{k}(t)+L_{c}(q) e_{k+1}(t)\right)
$$

where $L(q)$ is a linear, time-invariant, discrete-time, and possibly non-causal filter and $L_{c}(q)$ has to be a causal discrete-time filter. Furthermore $L_{c}(q)$ has to be chosen such that the transfer function of the closed loop system $1 /\left(1+L_{c}(q) T_{u}(q)\right)$ is asymptotically stable.

\subsection{Convergence criteria}

The algorithm properties will be studied by considering the update equation for the input signal. Combining equations (1) and (6) gives

$$
\boldsymbol{u}_{k+1}=\boldsymbol{F} \boldsymbol{u}_{k}+\boldsymbol{F}_{r} \boldsymbol{r}
$$

where

$$
\boldsymbol{F}=\left(I+\boldsymbol{Q} \boldsymbol{L}_{c} \boldsymbol{T}_{u}\right)^{-1} \boldsymbol{Q}\left(I-\boldsymbol{L} \boldsymbol{T}_{u}\right) \quad \boldsymbol{F}_{r}=\left(I+\boldsymbol{Q} \boldsymbol{L}_{c} \boldsymbol{T}_{u}\right)^{-1} \boldsymbol{Q}\left(\boldsymbol{L}_{c}+\boldsymbol{L}\right)
$$

In e.g. Norrlöf (2000) it is shown that a necessary and sufficient condition for convergence of the ILC algorithm is that $\rho(\boldsymbol{F})<1$, i.e. the largest eigenvalue of $\boldsymbol{F}$ is strictly less that one. For practical reasons it is of interest that the convergence of the input signal and the error is monotone. As shown in e.g. Norrlöf (2000) and Longman (2000) monotone convergence is obtained if the maximum singular value fulfills $\bar{\sigma}(\boldsymbol{F})<1$.

A frequency domain convergence condition is obtained by combining equations

(4) and (8). This gives the update equation for the input signal

$$
u_{k+1}(t)=\frac{Q(q)\left(1-L(q) T_{u}(q)\right)}{1+Q(q) L_{c}(q) T_{u}(q)} u_{k}(t)+\frac{Q(q)\left(L(q)+L_{c}(q)\right)}{1+Q(q) L_{c}(q) T_{u}(q)} r(t)
$$

and using this equation a frequency domain convergence conditions is found. 
The condition is given by

$$
\left|\frac{Q\left(e^{i \omega}\right)\left(1-L\left(e^{i \omega}\right) T_{u}\left(e^{i \omega}\right)\right)}{1+Q\left(e^{i \omega}\right) L_{c}\left(e^{i \omega}\right) T_{u}\left(e^{i \omega}\right)}\right|<1, \quad \forall \omega
$$

The singular value condition can be tied to a frequency domain conditions using the following results presented in e.g. Norrlöf (2000) and Longman (2000). Consider the transfer operator $F(q)$ associated with the matrix $\boldsymbol{F}$ in the sense that $\boldsymbol{F}$ is formed using the impulse response coefficients of $F(q)$. Assume that $F(q)$ is stable and causal. If $\left|F\left(e^{i \omega}\right)\right|<1 \quad \forall \omega$ then the largest singular value of $\boldsymbol{F}_{n}$ in the matrix representation of the system fulfills $\bar{\sigma}\left(\boldsymbol{F}_{n}\right)<1$ (where $\left.\boldsymbol{F}_{n} \in \mathbb{R}^{n \times n}\right)$.

\section{Time domain properties}

The observations below are based on some basic matrix properties. The first property is that the product of two lower triangular matrices having diagonal elements $\mu$ and $\lambda$ respectively is a lower triangular matrix with diagonal elements $\mu \cdot \lambda$. The second property is that the inverse of a lower triangular matrix having diagonal elements $\mu$ is a lower triangular matrix with diagonal elements $1 / \mu$. Consider now the matrix $\boldsymbol{F}$ defined in equation (10). Using the matrix properties above the following observations can be made.

\subsection{Causal ILC algorithms}

For $Q=I$, a causal ILC algorithm, i.e. $\boldsymbol{L}$ lower triangular, any choice of $\boldsymbol{L}_{c}$, and $g_{T_{u}}(0)=0$ no convergence can be achieved since since all the eigenvalues of $\boldsymbol{F}$ are equal to one. An exception is the special case $g_{T_{u}}(0) \neq 0$, i.e. that the system has relative degree zero. However, using $Q=\mu \cdot I$, where $0<\mu<1$ and a causal ILC algorithm, convergence to non-zero but arbitrarily small error can be achieved. 
The monotone convergence is determined by the largest singular value of $\boldsymbol{F}$, but using the connection between the singular value and the frequency function discussed in Section 2 this conditions can be changed to a frequency domain condition. This issue is discussed in Section 4, and it is shown that the monotone convergence condition, in most practical cases, can not be satisfied using a causal ILC algorithm.

\subsection{Non-causal ILC algorithms}

A main point of this paper is that the non-causal property of an ILC algorithm is the key to monotone convergence. There are several approaches to design of non-causal ILC algorithms. An experimental comparison of three different method is presented in Norrlöf and Gunnarsson (2002a).

Using an optimization approach, see e.g. Gunnarsson and Norrlöf (2001), one can design an ILC algorithm such that the singular value condition can be directly verified. An example is $\boldsymbol{L}_{c}=0, \boldsymbol{L}=\left(\lambda \cdot I+\boldsymbol{T}_{u}^{T} \boldsymbol{T}_{u}\right)^{-1} \boldsymbol{T}_{u}^{T}$, and $\boldsymbol{Q}=\rho \cdot I$. This gives that the singular values of $\boldsymbol{F}$ are given by

$$
\frac{\rho \cdot \lambda}{\lambda+\sigma_{i}^{2}}
$$

where $\sigma_{i}$ denote the singular values of $\boldsymbol{T}_{u}$. For any $\rho<1$, but arbitrarily close to one the condition of $\bar{\sigma}(\boldsymbol{F})<1$ is always satisfied.

Another example of a non-causal ILC algorithm is given in Section 5, where the design is carried out using the traditional frequency domain version of the monotone convergence condition. 


\section{Frequency domain properties}

Even though the frequency domain condition is only sufficient it is the condition that is of interest when it comes to practical use of ILC and algorithm design. The aim in this section is to give an intuitive understanding of the behavior of causal and CITE algorithms and to motivate why monotone convergence to zero error is impossible in most cases of practical interest.

\subsection{Bode's integral theorem}

Bode's integral theorem is a useful tool when understanding the properties of causal and CITE ILC algorithms. A discrete time formulation of this theorem can be found in e.g. Wu and Jonckheere (1992) and it can be stated as follows. Consider a discrete time system with transfer function

$$
F(z)=K \cdot \frac{\prod_{i=1}^{m}\left(z-z_{i}\right)}{\prod_{i=1}^{n}\left(z-p_{i}\right)}
$$

where $p_{i}$ 's are the open-loop poles, and some of them are being allowed outside the open-unit disc. Introduce the sensitivity function defined as

$$
S(z)=\frac{1}{1+F(z)}
$$

and assume that $K \neq 0$ is chosen such that $S(z)$ is asymptotically stable. Assume also that $T=1$. Then

$$
\int_{-\pi}^{\pi} \ln \left|S\left(e^{i \omega}\right)\right| d \omega=2 \pi \cdot\left(\sum_{i}\left|p_{u_{i}}\right|-\ln |\gamma+1|\right)
$$

where $p_{u_{i}}$ are the unstable poles and

$$
\gamma=\lim _{z \rightarrow \infty} F(z)
$$




\subsection{CITE ILC}

For the CITE ILC case the frequency domain convergence criterion, equation (12) becomes

$$
\left|1+L_{c}\left(e^{i \omega}\right) T_{u}\left(e^{i \omega}\right)\right|>1, \quad \forall \omega
$$

for the special case $Q\left(e^{i \omega}\right) \equiv 1$. In words the condition in equation (18) states that the curve $L_{c}\left(e^{i \omega}\right) T_{u}\left(e^{i \omega}\right)$ has to be outside a circle with unit radius and center in the point -1 . The circle is shown in Figure 1.

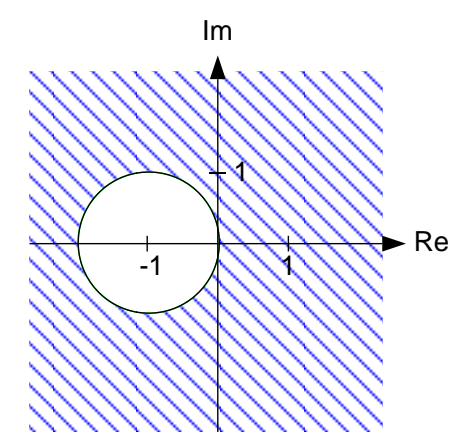

Fig. 1. Nyquist diagram interpretation of the convergence result in (18).

Using Bode's integral theorem it is straightforward to show that this condition holds only in a special case. Let the transfer function $F(z)$ in equation (14) be given by

$$
F(z)=L_{c}(z) T_{u}(z)
$$

where $T_{u}(z)$ is the transfer function of the system to be controlled and $L_{c}(z)$ is the causal filter in the ILC algorithm. For realistic systems $T_{u}(z)$ will have a relative degree that is at least one. Since $L_{c}(z)$ is a causal filter the relative degree is at least zero and hence the relative degree of $F(z)$ is at least one. This implies that $\gamma$, defined in equation (17), is zero under these conditions. If it is also assumed that both $L_{c}(z)$ and $T_{u}(z)$ are stable there are no $p_{u_{i}}$ 
(unstable poles) and hence

$$
\int_{-\pi}^{\pi} \ln \left|S\left(e^{i \omega}\right)\right| d \omega=0
$$

This result implies that if there is frequency range where $\left|S\left(e^{i \omega}\right)\right|<1$ i.e. $\ln \left|S\left(e^{i \omega}\right)\right|<0$ there has to be an interval where $\ln |S(i \omega)|>0$ which means

$$
\left|S\left(e^{i \omega}\right)\right|>1
$$

in order for the integral to be zero. The condition (21) can also be expressed

$$
\left|1+L_{c}\left(e^{i \omega}\right) T_{u}\left(e^{i \omega}\right)\right|<1
$$

which is a violation of the criterion in equation (18). The conclusion hence becomes that given the assumptions above there will always be an interval where the stability condition is violated. It will therefore be impossible to achieve monotone convergence to zero error in almost all cases of practical interest. It should also be noted that since the closed loop system that is formed using the CITE approach has to be stable the Nyquist stability criterion makes it impossible to choose $L_{c}(q)$ such that the curve $L_{c}\left(e^{i \omega}\right) T_{u}\left(e^{i \omega}\right)$ encircles the forbidden region.

In order to achieve monotone convergence for the CITE algorithm it is hence necessary to accept a non-zero final error. This is obtained by choosing the filter $Q(q)$ appropriately. Consider, for simplicity, $Q(q)=\rho$. The update equation is given by

$$
u_{k+1}(t)=\rho\left(u_{k}(t)+L_{c}(q) e_{k+1}(t)\right)
$$

which gives the modified convergence condition

$$
\left|1+\rho L_{c}\left(e^{i \omega}\right) T_{u}\left(e^{i \omega}\right)\right|>\rho, \quad \forall \omega
$$

Choosing $\rho<1$ reduces the radius of the circle that the Nyquist curve has to be outside. At the same time the Nyquist curve itself is scaled by $\rho$ and 
this also makes it easier to satisfy the convergence condition. It is important to note that $\rho$ has to be chosen sufficiently small to satisfy equation (24). Consequently the final error can not be made arbitrarily small at the same time as monotone convergence is obtained.

\subsection{Causal classical ILC}

Using a causal classical ILC algorithm the convergence condition becomes

$$
\left|1-L_{o}\left(e^{i \omega}\right) T_{u}\left(e^{i \omega}\right)\right|<1, \quad \forall \omega
$$

in the special case $Q\left(e^{i \omega}\right) \equiv 1$. The interpretation of this condition is that the curve $L_{o}\left(e^{i \omega}\right) T_{u}\left(e^{i \omega}\right)$ has to be inside the so called learning circle, which has unit radius and is centered in one.

By putting $F(z)=L(z) T_{u}(z)$ and assuming that the relative degree of $T_{u}(z)$ is at least one, Bode's integral theorem implies that there will always be a frequency interval where $\left.L_{(} e^{i \omega}\right) T_{u}\left(e^{i \omega}\right)$ is inside the circle shown in Figure 1. If the curve is inside the circle in Figure 1 it is obvious that it will be outside the learning circle, and hence the convergence criterion is violated. The conclusion is that monotone convergence to zero error using a causal ILC algorithm can not be obtained in general.

Also here the convergence condition can be relaxed by allowing a non-zero error. For simplicity consider the case $Q(q)=\rho$ where $0<\rho<1$ The convergence condition then becomes

$$
\left|1-L\left(e^{i \omega}\right) T_{u}\left(e^{i \omega}\right)\right|<1 / \rho, \quad \forall \omega
$$

This means that the radius of the learning circle is increased and the criterion is more easily satisfied. For the classical ILC algorithm the second and fundamentally different alternative compared to the CITE algorithm is to use a non-causal filter $L(q)$. This will be illustrated in the example in Section 5 . 


\section{Illustrative example}

To support the discussion in the previous section an illustrative example is given. First assume that the system under consideration has the following form,

$$
T_{u}(q)=\frac{q}{q^{2}-0.5 q+0.5}
$$

i.e., a second order system with relative degree one. This is a discrete time version of a continuous time two-mass system coupled by a spring and damper system.

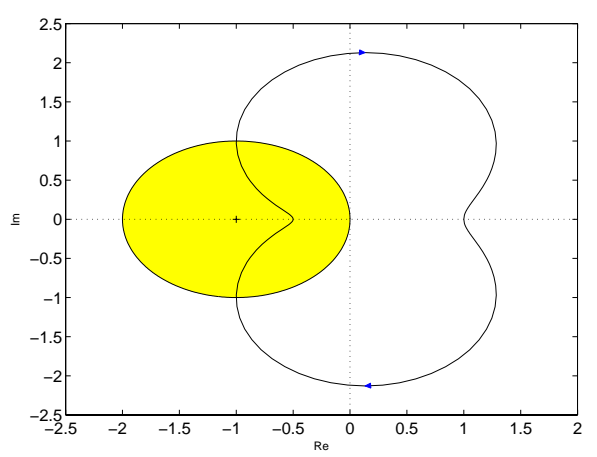

Fig. 2. Nyquist diagram for the system $T_{u}(q)$ in (27).

First consider the case when the system in (27) is controlled by a CITE ILC algorithm. In Figure 2 the Nyquist diagram for $T_{u}$ from (27) is depicted. It is clear that the frequency domain criterion for CITE in (18) does not hold in this case (when $L_{c}(q)=1$ ). Also using a causal ILC according to Section 4.3 will not give an ILC algorithm that fulfills the condition in (25) (when $L(q)=1)$. Assume that $L_{c}(q)$ is restricted to be a scalar. Clearly it is possible to fulfill (18) by just letting $\gamma$ in $L_{c}(q)=\gamma$ take a large enough value. The Nyquist plot of $L_{c}\left(e^{i \omega}\right) T_{u}\left(e^{i \omega}\right)$ then will encircle the forbidden region in the left half plane, see Figure 2. The problem with this approach is however that -1 is inside the forbidden region and since it is encircled by the Nyquist plot of $L_{c}\left(e^{i \omega}\right) T_{u}\left(e^{i \omega}\right)$ the closed loop will be unstable. 
Following the ideas in Section 4 actually gives a stronger result since, according to the result based on the Bode's integral theorem, there is no CITE ILC algorithm or causal classical ILC algorithm that will satisfy the frequency domain conditions. This follows from the fact that the system in (27) has relative degree 1 .

A common choice in classical ILC algorithms is to choose the non-causal filter $L(q)=\gamma q^{\delta}$ where $\delta>0$ is the time delay of the system and $\gamma$ is a gain that can be decided. In Figure 3 Nyquist diagrams of $L\left(e^{i \omega}\right) T_{u}\left(e^{i \omega}\right)$ for two choices of $L(q)$ are shown. In both cases $\delta=1$ while $\gamma=1$ in the first case (solid) and $\gamma=0.75$ in the second case (dashed). Clearly the second choice fulfills the frequency domain condition in (25) while the first does not. As was pointed out in Section 2.2 the frequency domain condition is only a sufficient condition while a necessary and sufficient condition is that the largest eigenvalue of $\boldsymbol{F}=I-\boldsymbol{L} \boldsymbol{T}_{u}$ is strictly less than one. With the system given in $(27)$ and the size of the matrices $25 \times 25$ the resulting largest eigenvalues become $\rho(\boldsymbol{F})=0$ when $\gamma=1$ and $\rho(\boldsymbol{F})=0.25$ when $\gamma=0.75$. This shows that the ILC algorithm is stable in both cases. If the largest singular value $\bar{\sigma}(\boldsymbol{F})$ is computed instead, the results become $\bar{\sigma}(\boldsymbol{F})=1.18$ when $\gamma=1$ and $\bar{\sigma}(\boldsymbol{F})=0.79$ when $\gamma=0.75$. In the first case monotone convergence of the error cannot be guaranteed while in the second case it can be guaranteed (as shown in Figure 3).

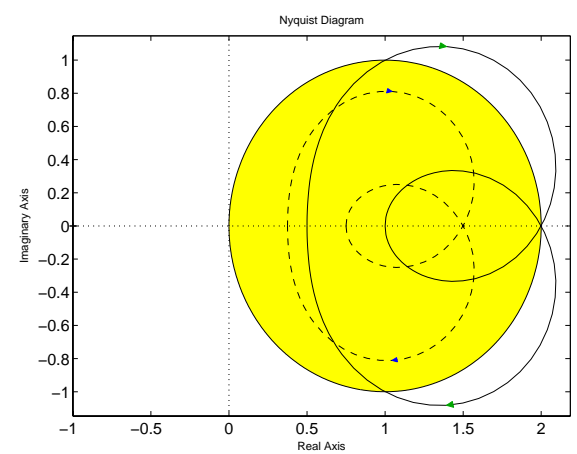

Fig. 3. Nyquist diagram of $L(q) T_{u}(q)$ when $L(q)=q$ (solid) and $L(q)=0.75 q$ (dashed). 


\section{Conclusions}

Time and frequency domain convergence conditions for causal ILC algorithms have been studied. A necessary and sufficient convergence condition is expressed in terms of an eigenvalue condition. For practical use it is however more important to consider a condition for monotone convergence. This can be expressed as a singular value condition or a frequency domain condition. It is claimed that the key to monotone convergence of ILC algorithms is to use non-causal operators in the algorithm. It has been shown that causal ILC algorithms in general and CITE ILC algorithms in particular will in general not be able to converge monotonously to zero error. This property has been shown by using a discrete time version of Bode's integral theorem. The results have been supported using a numerical example.

\section{Acknowledgment}

This work was supported by ISIS and CENIIT at Linköping University.

\section{References}

Arimoto, S., Kawamura, S., Miyazaki, F., 1984. Bettering operation of robots by learning. Journal of Robotic Systems 1 (2), 123-140.

Chen, Y., Xu, J.-X., Lee, T. H., Dec 1996a. Current iteration tracking error assisted iterative learning control of uncertain nonlinear discrete-time systems. In: Proc. of the 35th IEEE Conf. on Decision and Control. Kobe, Japan.

Chen, Y., Xu, J.-X., Lee, T. H., Dec 1996b. An iterative learning controller using current iteration tracking error information and initial state learning. In: Proc. of the 35th IEEE Conf. on Decision and Control. Kobe, Japan. 
French, M., Munde, G., Rogers, E., Owens, D., Dec 1999. Recent developments in adaptive iterative learning control. In: Proc. of the 38th IEEE Conference on Decision and Control. Pheonix, Arizona, USA.

Goldsmith, P. B., April 2002. On the equivalence of causal lti iterative learning control and feedback control. Automatica 38, 703-708.

Gunnarsson, S., Norrlöf, M., 2001. On the Design of ILC Algorithms Using Optimization. Automatica 37, 2011-2016.

Horowitz, R., June 1993. Learning control of robot manipulators. Journal of Dynamic Systems, Measurement, and Control 115, 402-411.

Lange, F., Hirzinger, G., May 1999. Learning accurate path control of industrial robots with joint elasticity. In: Proc. IEEE Conference on Robotics and Automation. Detriot, MI, USA.

Longman, R., July 2000. Iterative learning control and repetitive control for engineering practice. International Journal of Control 73 (10), 930 - 954.

Norrlöf, M., Gunnarsson, S., 2002a. Experimental comparison of some classical iterative learning control algorithms. IEEE Transactions on Robotics and Automation 18, 636-641.

Norrlöf, M., Gunnarsson, S., September 2002b. Some new results on current iteration trackin error ilc. In: Asian Control Conference. Singapore.

Norrlöf, M., 2000. Iterative learning control: Analysis, design, and experiments. Ph.D. thesis, Linköpings universitet, Linköping, Sweden, linköping Studies in Science and Technology. Dissertation 653. Download from http://www.control.isy.liu.se/publications/.

Wu, B.-F., Jonckheere, E. A., 1992. A simplified aproach to bode's theorem for continuous-time and discrete-time systems. IEEE Transactions on Automatic Control 37, 1797-1802. 